\title{
ARTICLE Ventral striatal response during decision making involving risk and reward is associated with future binge drinking in adolescents
}

\author{
Angelica M. Morales ${ }^{1}$, Scott A. Jones ${ }^{1}$, Alissa Ehlers ${ }^{2}$, Jessye B. Lavine ${ }^{2}$ and Bonnie J. Nagel ${ }^{1,2}$
}

\begin{abstract}
Beginning to engage in heavy alcohol use during adolescence, as opposed to later in life, is associated with elevated risk for a variety of negative consequences, including the development of an alcohol use disorder. Behavioral studies suggest that poor decision making predicts alcohol use during adolescence; however, more research is needed to determine the neurobiological risk factors that underlie this association. Using functional magnetic resonance imaging, brain activation during decision making involving risk and reward was assessed in 47 adolescents (14-15 years old) with no significant history or alcohol or drug use. After baseline assessment, participants completed follow-up interviews every 3 months to assess the duration to onset of binge drinking. Adolescents who made a greater number of risky selections and had greater activation in the nucleus accumbens, precuneus, and occipital cortex during decision making involving greater potential for risk and reward began binge drinking sooner. Findings suggest that heightened activation of reward circuitry during decision making under risk is a neurobiological risk factor for earlier onset of binge drinking. Furthermore, brain activation was a significant predictor of onset to binge drinking, even after controlling for decision-making behavior, suggesting that neurobiological markers may provide additional predictive validity over behavioral assessments. Interventions designed to modify these behavioral and neurobiological risk factors may be useful for curbing heavy alcohol use during adolescence.
\end{abstract}

Neuropsychopharmacology (2018) 43:1884-1890; https://doi.org/10.1038/s41386-018-0087-8

\section{INTRODUCTION}

Alcohol use is prevalent during adolescence [1] and is associated with long-term negative consequences (for a review, see [2]). The percentage of adolescents reporting past-month alcohol use increases from $\sim 7$ to $33 \%$ between eighth and 12th grade [1], and $\sim 33 \%$ of young drinkers transition to binge drinking within 12 months of their first drink [3]. Furthermore, individuals who first experience alcohol intoxication at an earlier age are at heighted risk for future problems, such as risky alcohol or substance use [4, 5], drunken driving [5], and risky sexual behavior [6]. Identifying the behavioral and neurobiological risk factors that predict binge drinking during adolescence may be useful for identifying highrisk individuals and developing interventions to curb or delay heavy alcohol use during this developmentally sensitive epoch.

Longitudinal studies have demonstrated that poor decision making in situations involving uncertainty predicts future alcohol use during adolescence and early adulthood. Using the lowa Gambling Task, a study of 15-16 year olds demonstrated that a higher proportion of disadvantageous selections was associated with binge drinking 1 year later [7]. Similarly, college students who maintain high levels of binge drinking over the course of 2 years made a greater proportion of disadvantageous selections on the lowa Gambling Task during baseline assessments than students who engaged in low levels of binge drinking over the same time period [8]. Since optimal performance on the lowa Gambling Task relies on the ability to learn uncertain risk and reward probabilities, it is difficult to determine whether these findings reflect learning impairments or individual differences in risk/ reward sensitivity. Additional research is necessary to determine whether decision making in situations where levels of risk and reward are made explicit also predict future alcohol use. Further, these studies included participants with prior alcohol exposure; therefore, it remains unclear whether poor decision making is a premorbid risk factor for binge drinking or a consequence of early alcohol use.

The neural circuitry implicated in decision making involving risk and reward undergoes development during adolescence (for a review, see [9]). Functional magnetic resonance imaging (MRI) studies have demonstrated that adolescents have greater brain activation in the ventral striatum during reward processing than children or adults [10-12], suggesting that adolescents experience heightened reward sensitivity. Furthermore, cognitive control increases throughout adolescence [13], a process which is accompanied by the protracted maturation of the prefrontal cortex $[14,15]$. It has been hypothesized that this imbalance between bottom-up reward and top-down control processes may result in risky choices, such as the decision to engage in heavy alcohol use [16]. Consistent with this hypothesis, a previous study found that largely alcohol-naïve adolescents who went on to engage in binge drinking had less activation in prefrontal, parietal,

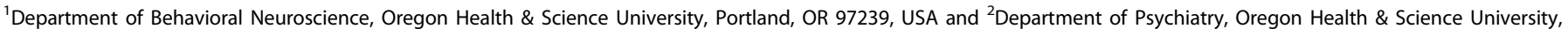
Portland, OR 97239, USA

Correspondence: Bonnie J. Nagel (nagelb@ohsu.edu)

Received: 11 January 2018 Revised: 23 April 2018 Accepted: 29 April 2018

Published online: 7 May 2018 
and temporal cortices during risky compared to safe decision making than adolescents who did not subsequently engage in binge drinking [17]. However, since this study examined brain activation as a function of decision-making behavior, adolescents who made mostly risky or safe selections were excluded from the analyses. Examination of brain activation during decision making under varying levels of risk/reward, irrespective of decisionmaking behavior, may be useful for characterizing the full range of individual differences in neurobiology that contribute to task performance and real-world engagement in risky behavior.

To identify premorbid risk factors for onset of heavy drinking, in the current study, we measured brain activation during decision making involving known levels of risk and reward in adolescents with minimal history of alcohol or drug use. Baseline assessments were conducted when participants were between the ages of 14 and 15, the developmental time period associated with the greatest functional imbalance between reward and cognitive control circuitry (for a review, see [9]). Follow-up interviews were conducted every 3 months to assess the emergence of alcohol use. Based on previous findings [7, 8], we hypothesized that adolescents who made a greater proportion of risky selections at baseline would begin binge drinking sooner. Furthermore, we anticipated that greater activation in brain regions implicated in reward processing and less activation in brain regions implicated in cognitive control would be associated with risky decision making and shorter duration to onset of binge drinking.

\section{MATERIALS AND METHODS}

Participant characterization and study design

Participants were 47 healthy adolescents between the ages of 14 and 15 who were recruited from the community as part of an ongoing study of adolescent neurodevelopment [17-19], which was approved by the Oregon Health \& Science University (OHSU) Institutional Review Board. Exclusionary criteria for youth included left handedness (Edinburgh Handedness Inventory [20]), DSM-IV psychiatric diagnoses [21], serious medical problems, significant head trauma, mental retardation or learning disabilities, psychotic illness in a biological parent (e.g., schizophrenia or bipolar I), parent-reported prenatal exposure to drugs or alcohol, MRI contraindications (e.g., irremoveable metal in the body), or pregnancy. At baseline, participants had to be free of any significant alcohol or drug use. Specifically, participants were excluded if they reported consuming $>10$ lifetime alcoholic drinks, $>2$ alcoholic drinks on any one occasion, $>10$ lifetime uses of marijuana, $>4$ lifetime uses of cigarettes, or any other drug use (Customary Drinking and Drug Use Record [22]).

At baseline, intellectual functioning was assessed using the twosubtest form of the Wechsler Abbreviated Scale of Intelligence [23], socioeconomic status was estimated using parent self-report with the Hollingshead Index of Social Position [24], pubertal development was measured using participant self-report on the Pubertal Development Scale [25], and sensation seeking was measured using the Impulsive Sensation Seeking Scale of the Zuckerman-Kuhlman Personality Questionnaire [26]. To assess decision making in situations involving risk and reward, participants completed a modified version of the Wheel of Fortune (WOF) Task [19, 27] paired with functional MRI (details below).

After completion of the baseline study procedures, participants completed follow-up phone interviews approximately every 3 months. During these interviews, participants completed the Customary Drinking and Drug Use Record and the 90-day Timeline Followback [28] to assess any changes in substance use. If participants missed a follow-up assessment, a longer version of the Timeline Followback was administered to capture all substance use information. Participants are part of an ongoing study and will be followed through age 21 . We calculated the number of months before participants began binge drinking, where binge drinking was defined as $\geq 5$ drinks per occasion for males and $\geq 4$ drinks per occasion for females (based on criteria established by the National Institute on Alcohol Abuse and Alcoholism [29]).

\section{WOF task}

During this computerized two-choice decision-making task (Supplemental Fig. 1), participants made choices under situations where the difference in risk/reward between available options was high $(10 \%$ probability of winning $\$ 7$ versus $90 \%$ probability of winning $\$ 1)$, moderate $(30 \%$ probability of $\$ 2$ versus $70 \%$ probability of winning $\$ 1$ ), or equal (50/50 chance of winning \$2). The potential for risk and reward associated with competing options was made explicit using a wheel divided into two portions based on the probability of winning distinct dollar amounts. Trials were $10.5 \mathrm{~s}$ long and consisted of selection ( $3 \mathrm{~s})$, anticipation (3.5 s), and feedback (4s) phases, with intertrial fixation intervals jittered between 1-11 s. During the selection phase, participants were instructed to select the portion of the wheel they thought would win them the greatest amount of money, as they would receive a portion of their total earnings. During the anticipation phase, participants indicated how sure they were of winning on a scale of $1-3$. Following the anticipation phase, participants received feedback via a screen that indicated whether they won or not alongside their cumulative earnings. While in the scanner, adolescents performed 72 trials, presented during two 10-min runs of 36 trials each. Each run included 12 high risk/reward wheels, 14 moderate risk/reward wheels, and 10 equal risk/reward wheels. Given our current interest in how decision making may influence the initiation of binge drinking, we examined the brain activation during the selection phase.

\section{Scan acquisition}

Participants were scanned at OHSU's Advanced Imaging Research Center on a 3.0 Tesla Siemens Magnetom Tim Trio. Anatomical, high-resolution T1-weighted MPRAGE structural scans were collected in the sagittal plane $(T R=2300 \mathrm{~ms}, T E=3.58 \mathrm{~ms}$, inversion time $=900 \mathrm{~ms}$, flip angle $=10^{\circ}$, voxel size $=1 \times 1 \times 1.1$ $\mathrm{mm}$ ). Blood-oxygen-level dependent (BOLD) signal was measured using $\mathrm{T}^{*}$ weighted gradient echo-planar images which were collected axially, parallel to the anterior-posterior commissure line $\left(\mathrm{TR}=2000 \mathrm{~ms}, \mathrm{TE}=30 \mathrm{~ms}\right.$, flip angle $=90^{\circ}$, voxel size $=3.75 \times$ $3.75 \times 3.8 \mathrm{~mm}$ ).

\section{Functional MRI preprocessing}

After initial visual inspection for artifacts in BOLD images, Analysis of Functional Neurolmages [30] was used to conduct slice time correction and estimate alignment all of the TRs to the volume requiring the least amount of adjustment. To minimize interpolation error, spatial transformations to adjust for motion, co-register the BOLD images to the high-resolution structural image, and conduct nonlinear registration of the high-resolution structural image to a standard template in Talairach space were combined and applied to BOLD images in a one-step registration process. Finally, the images were smoothed using a 6-mm Gaussian kernel and percent signal change was calculated for each run.

The first-level model included regressors representing the selection, anticipation, and feedback trials. Stimulus times corresponding to the onset time of each phase and the duration of the event coded as the length of each phase were convolved with a gamma-variate hemodynamic response function [31]. Additional regressors modeled linear drift and motion parameters. Volumes were censored if framewise displacement exceeded 0.7 $\mathrm{mm}$ or if they were part of uncensored segments of data with fewer than five contiguous frames, based on prior research demonstrating that motion scrubbing thresholds for task-based functional MRI are most effective within the range of $0.5-1.1 \mathrm{~mm}$ [32]. Contrast images of average percent signal change between 

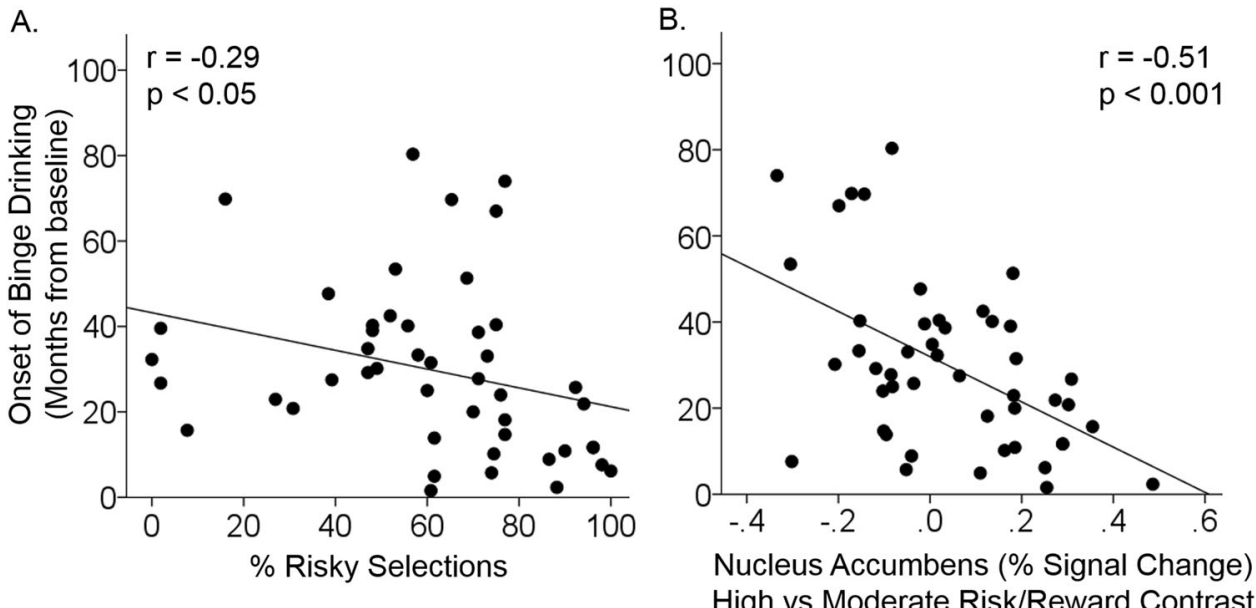

Fig. 1 Associations between risky decision making, brain activation, and duration to onset of binge drinking. Scatter plots show that adolescents who made a greater proportion of risky selections (a) and who had greater nucleus accumbens activation during decision making involving high versus moderate levels of risk/reward (b) started binge drinking sooner

high and moderate levels of risk/reward during the selection phase were resampled to $3 \mathrm{~mm}^{3}$ voxels prior to group-level analyses.

\section{Group analyses}

All voxel-wise group-level analyses used a cluster-forming threshold of $p<0.01$ and results were corrected for multiple comparisons using a nonparametric permutation analysis (3dttest ++ -Clustsim), which determined the number of voxels needed to achieve cluster-level significance at a two-sided threshold of $p<$ 0.05 [33]. While a recent study found inflated false-positive rates using parametric statistical methods [34], the nonparametric approach used in this study provides adequate control over false positives at a cluster-forming threshold of $p<0.01$ [33]. A onesample $t$-test was used to examine changes in brain activation during decision making under high versus moderate risk/reward conditions (cluster extent $>234$ voxels). Since the bilateral nucleus accumbens is a small brain region $\left(71,3-\mathrm{mm}^{3}\right.$ voxels in Talairach space) of a priori interest, brain activation in this region was examined by extracting percent signal change from a mask defined using the Harvard-Oxford Atlas (Supplemental Fig. 2). Bivariate correlations were used to assess the relationships between nucleus accumbens activation, proportion of risky selections, and duration to onset of binge drinking. Whole-brain linear regression analyses were also conducted to examine the association between activation in the high versus moderate risk/ reward contrast and proportion of risky selections (cluster extent $>273$ voxels) and duration to onset of binge drinking (cluster extent $>230$ ). Post hoc analyses in the nucleus accumbens and across the whole brain examined whether significant associations could be explained by brain activation in the high versus equal or moderate versus equal risk/reward contrasts.

\section{RESULTS}

Participant characteristics

Twenty-eight females and 19 males were included in the study. On average, participants were $15.07(S D=0.59)$ years old, had IQ scores of $111.02(S D=9.16)$, and scored $28.62(S D=13.06)$ on the Hollingshead Index of Social Position. Since none of these demographic variables were significantly associated with duration to onset of binge drinking ( $p^{\prime} s>0.05$ ), they were not included as covariates in subsequent analyses. As anticipated based on exclusion criteria, participants reported $0.36(S D=1.07)$ instances of alcohol use, $0.68(S D=1.83)$ instances of marijuana use, and
$0.06(S D=0.32)$ instances of cigarette use in their lifetime. Although the rates of lifetime substance use were very low, analyses were also conducted in participants who were completely alcohol and drug naïve at baseline $(n=34,23$ female/11 male). On the WOF Task, participants chose the risky option approximately $60.61 \%$ of the time $(S D=26.21)$ with similar proportions of risky selections being made on high (57.65 \pm $30.35)$ and moderate $(63.01 \pm 27.02)$ risk/reward trials $(t(46)=$ $-1.58, p=0.12$ ). There were no significant associations between proportion of risky selections and sex, age, IQ scores, or the Hollingshead Index of Social Position ( $p^{\prime} s>0.05$ ). Based on followup assessments, participants began binge drinking 29.88 months $(\mathrm{SD}=19.82)$ after baseline assessments.

Association between decision-making behavior and onset of binge drinking

Adolescents who made a greater proportion of risky selections began binge drinking sooner $(r=-0.29, p<0.05$, Fig. 1a). Followup analyses to determine whether this association was driven by risky selections in high $(r=-0.24, p=0.10)$ or moderate $(r=$ $-0.29, p<0.05)$ risk/reward conditions revealed a similar effect in both conditions. However, none of these associations were significant in participants who were completely alcohol and drug naïve at baseline ( $\left.p^{\prime} s>0.05\right)$.

Association between brain activation and onset of binge drinking Differences in brain activation between the high and moderate risk/reward contrast were detected in the bilateral medial and lateral prefrontal cortex, anterior insula, striatum, and the left cuneus (Fig. 2). Region-of-interest analysis revealed that adolescents with greater nucleus accumbens activation in the high versus moderate risk/reward contrast began binge drinking sooner ( $r=-0.51, p<0.001$, Fig. $1 \mathrm{~b}$ and Supplemental Fig. 3). These effects were driven, in part, by a negative association between duration to onset of binge drinking and brain activation in bilateral nucleus accumbens in the high versus equal risk/ reward contrast $(r=-0.32, p<0.05)$, but no association between duration to onset of binge drinking and brain activation in the moderate versus equal risk/reward contrast $(r=0.19, p>0.1)$. Furthermore, whole-brain analysis revealed that adolescents who began binge drinking sooner also had greater activation in left fusiform gyrus, right precuneus, and right middle occipital gyrus in the high versus the moderate risk/reward condition $(p<0.05$ corrected, Table 1, Fig. 3, Supplemental Figure 4). Post hoc wholebrain analyses did not reveal any significant associations between 


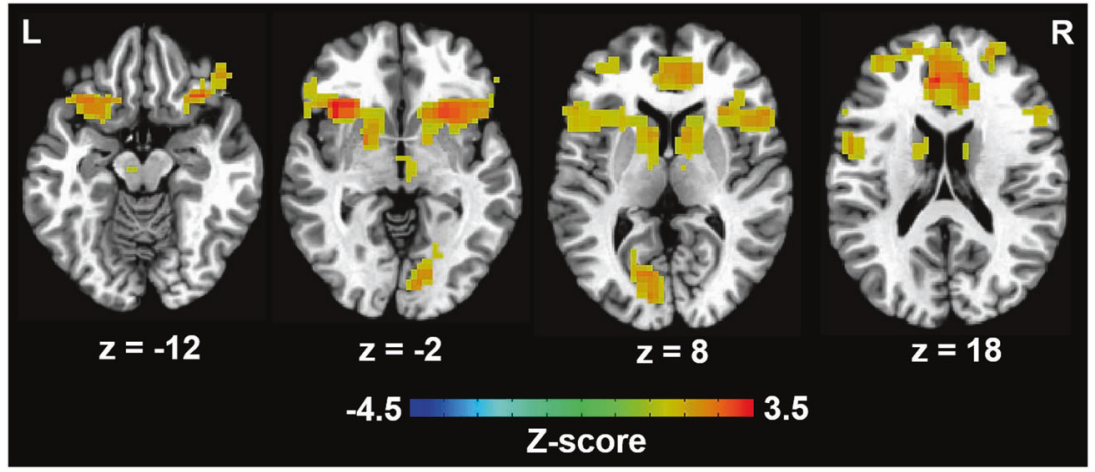

Fig. 2 Changes in brain activation during decision making involving high versus moderate levels of risk and reward. On average, adolescents displayed greater brain activation in bilateral medial and lateral prefrontal cortex, anterior insula, striatum, and left cuneus during decision making involving high versus moderate levels of risk and reward ( $p<0.05$, corrected for multiple comparisons)

duration to onset of binge drinking and brain activation in high versus equal or moderate versus equal risk/reward contrasts. The associations between duration to onset of binge drinking and brain activation in the nucleus accumbens $(r=-0.63, p<0.001)$, left fusiform gyrus, right precuneus, and right middle occipital gyrus ( $p<0.05$ corrected) during decision making in the high versus moderate risk/reward contrast remained significant in the sample of adolescents who were completely drug and alcohol naïve at baseline.

Behavioral and neural prediction of duration to onset of binge drinking

No significant associations were detected between proportion of risky selections and brain activation in the high versus moderate risk/reward contrast ( $\left.p^{\prime} s>0.05\right)$. To determine whether behavior and neural activation were independent predictors of duration to onset of binge drinking, behavior and nucleus accumbens activation were included as predictors in the same statistical model. The nucleus accumbens was chosen because the region was of a priori interest, and including additional brain regions in the model would have resulted in multicollinearity. When included in the same statistical model $\left(R^{2}=0.34, F(2,44)=11.54 p<0.001\right)$, proportion of risky selections $(\beta=-0.30, p<0.05)$ and bilateral nucleus accumbens $(\beta=-0.51, p<0.001)$ activation in the high versus moderate risk/reward contrast were both significant predictors of duration to onset of binge drinking. The statistical model including nucleus accumbens activation as a predictor performed better than the model that only included decisionmaking behavior $\left(R^{2}\right.$-change $\left.=0.26, F(1,44)=17.41, p<0.001\right)$.

Finally, we examined the extent to which our findings may be influenced by sex or sensation seeking. When including sex as covariate $(\beta=-0.10, p>0.05)$, nucleus accumbens activation in the high versus moderate risk/reward condition $(\beta=-0.50, p<$ $0.001)$ and risky selections $(\beta=-0.30, p<0.05)$ remained significant predictors of duration to onset of binge drinking. Sensation seeking was positively correlated with risky selections $(r=0.32, p=0.31)$, but was not significantly associated with duration to onset of binge drinking $(r=-0.24, p>0.05)$ or nucleus accumbens activation in the high versus moderate risk/reward condition $(r=-0.06, p>0.05)$. Duration to onset of binge drinking remained significantly associated with nucleus accumbens activation in the high versus moderate risk/reward condition $(\beta=-0.52$, $p<0.001)$ when including risky selections $(\beta=-0.23, p=0.07)$ and sensation seeking as covariates $(\beta=-0.20, p>0.10)$.

\section{DISCUSSION}

This prospective study provides evidence that duration to onset of binge drinking in adolescence can be predicted by behavioral performance and brain activation during a laboratory-based assessment of decision making involving risk and reward. Although the association between risky decision-making behavior and future binge drinking was relatively small, neural markers were better predictors, with nucleus accumbens activation accounting for $\sim 25 \%$ of the variance in duration to onset of binge drinking. Furthermore, this effect remained significant in adolescents who were completely alcohol and drug naïve at baseline ( $40 \%$ of variance explained), suggesting that heightened brain activation during decision making involving greater potential for risk and reward represents a premorbid risk factor for earlier onset of heavy alcohol use.

Findings from preclinical studies suggest that the nucleus accumbens plays a critical and complex role in value-based decision-making behavior. These studies have shown that lesion or inactivation of the nucleus accumbens increases risk-taking behavior [35, 36], that dopamine release in the nucleus accumbens during decision making codes subjective reward preference [36], and that activation of nucleus accumbens neurons during decision making reflects prior outcomes [37]. Although we did not find an association between nucleus accumbens activation and task-performance, we did find that greater nucleus accumbens activation predicted earlier engagement in binge drinking. Consistent with the preclinical literature, these findings suggest that nucleus activation during choice evaluation, prior to learning the reward outcome of the current decision, plays an important role in guiding behavior. More work is necessary to disentangle the factors that contribute to nucleus accumbens activation during decision making on the WOF Task and to determine whether nucleus accumbens activation during decision making can predict engagement in other real-world risky behaviors that are common during adolescence.

Our findings extend prior work indicating that nucleus accumbens responsivity is an important predictor of future substance use. A previous study demonstrated that less activation in the nucleus accumbens during reward anticipation at age 14 predicted problematic drug use by age 16 [38]. In contrast, we found that greater nucleus accumbens activation during decisions involving greater potential for risk and reward was associated with earlier engagement in binge drinking. The opposing direction of these findings could be attributable to differences in the experimental paradigms used to elicit striatal activation. Büchel and colleagues measured reward anticipation using the Monetary Incentive Delay (MID) Task, a reaction-time paradigm involving no decision making or risk. Prior studies have demonstrated that compared with adults, adolescents show less activation in the ventral striatum during the MID, but greater ventral striatal activation during tasks involving decision making and risk (for a review, see [9]). Furthermore, Büchel and colleagues only found a significant association between nucleus accumbens activation and future engagement in problematic drug use in adolescents who 
Table 1. Whole-brain analysis of the association between duration to onset of binge drinking and brain activation in high versus moderate risk/ reward contrasts

Brain regions (location of peak listed first)

Hemisphere

\begin{tabular}{llll}
\multicolumn{2}{l}{ Talairach coordinates } & & $\begin{array}{l}\text { Cluster extent } \\
\text { (voxels) }\end{array}$ \\
\cline { 2 - 3 } & $y$ & $z$ & \\
40 & 55 & -14 & 722 \\
-22 & 70 & 25 & 507 \\
-49 & 70 & -5 & 348
\end{tabular}

\begin{tabular}{l} 
Z-score \\
\hline-4.53 \\
-4.55 \\
-4.53
\end{tabular}

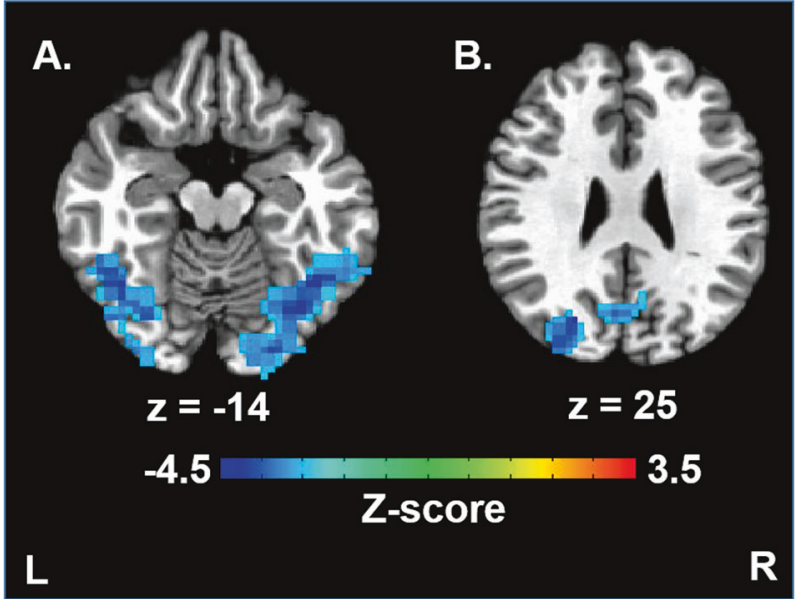

Fig. 3 Onset of binge drinking behavior is associated with brain activation during decision making involving risk and reward. Wholebrain voxel-wise statistical maps show brain regions where greater activation during decision making involving high versus moderate levels of risk and reward were associated with duration to onset of binge drinking. Duration to onset of binge drinking was also associated with brain activation in the left fusiform gyrus (a), right precuneus (b), and right middle occipital/fusiform gyri (a) (clusterforming threshold $p<0.01$, cluster-level corrected for multiple comparisons at $p<0.05$ )

scored in the top quartile on an assessment of novelty seeking [38]. In the current study, nucleus accumbens activation in the high risk/reward condition, but not the moderate risk/reward condition, was associated with duration to onset of binge drinking. Together, these findings suggest that ventral striatal activation in situations involving high levels of potential risk and reward represents a more sensitive or ecologically valid assessment of individual differences in susceptibility for future drug use across all adolescents.

As brain activation in the fusiform gyrus and precuneus also predicted duration to onset of binge drinking, developing a better understanding of the role these regions play in decision making may be useful for predicting the risk for heavy drinking. A metaanalysis of existing functional MRI studies demonstrated that decision making is consistently associated with activation of the fusiform gyrus and precuneus [39]; however, the fusiform gyrus was more likely to be active during decision making involving risk, while the precuneus was more likely to be active during decision making involving ambiguity. A study attempting to dissociate the effect of stimulus value and salience during decision making demonstrated that brain activation in the fusiform gyrus was correlated with stimulus salience, while activation in the precuneus was correlated with stimulus value [40]. As risk and potential reward value were known in the current task, this could help to explain why activation in both regions was implicated in predicting future alcohol use. Furthermore, research is needed to determine whether abnormal patterns of brain activation in these regions also predict other drug use. A cross-sectional study showed that adolescents with cannabis use disorder had more activation in precuneus during decision making involving risk than a control group [41], suggesting that heightened precuneus activation during decision making may reflect a premorbid risk factor for substance use, more generally.

A variety of biological and environmental factors may impact individual differences in neurobiological responses during decision making involving risk and reward. For example, genetic variants thought to influence dopamine signaling have been linked to brain activation during decision making [42] and in response to reward processing $[43,44]$. Epigenetic factors may also contribute to individual differences in risk/reward processing, as a recent study showed that DNA methylation of the promoter for the dopamine transporter gene was associated with nucleus accumbens activation during loss anticipation [45]. There is also evidence that environmental factors can influence reward-related brain activation. For example, studies examining risky decision making and associated brain activation using a simulated driving task have shown that adolescents make fewer risky decisions and show lower associated ventral striatal activation when their mother was present [46], as well as greater risky decisions and associated ventral striatal response in the presence of peers [47]. These studies highlight how social context further shapes decision making and neurobiological response during adolescence and suggest that future studies examine how these influences may further inform subsequent behavior, such as engagement in binge drinking.

Though novel, this work is not without limitations. The task design used in this study precludes examination of how risk and reward sensitivity independently modulate brain activation and whether sensitivity to each of these factors influences task performance and future alcohol use. This limitation may explain why we did not detect associations between brain activation and the proportion of risky selections, as greater reward and risk sensitivity may have opposing effects on behavior that could not be dissociated. Furthermore, since other studies have demonstrated that brain activation in reward circuitry tracks potential for both gains and losses during decision making [48], future studies could examine if behavior and associated brain activation in situations involving potential for both gains and losses would produce similar or distinct patterns of results. Another limitation of this study is that participants only underwent testing once before the initiation of binge drinking. Previous studies have shown that although ventral striatal activation in response to reward peaks in adolescence, there are significant individual differences in this effect [12]. Assessing adolescents at regular intervals before the initiation of substance use may be useful for determining the extent to which risky decision making and associated neurobiological responses represent stable or transient windows of increased risk for substance use.

These findings contribute to our understanding of the premorbid neurobiological risk factors for earlier engagement in binge drinking, behavior which may result in a variety of longterm negative consequences. Preclinical studies have demonstrated that the long-term consequences associated with binge 
drinking differ based on the timing of exposure (for a review, see [2]). For instance, one study demonstrated that rats exposed to binge-like levels of alcohol during early adolescence displayed greater motivation for alcohol self-administration in adulthood than rats exposed to alcohol during late adolescence [49]. These differences in behavior were accompanied by differences in c-Fos immunoreactivity, a marker of neuronal activity, in the nucleus accumbens in adulthood, suggesting that alcohol exposure may be particularly deleterious during certain developmental periods. Taken together, these studies suggest that interventions that provide adolescents with the tools to regulate ventral striatal responsivity in particular contexts have the potential to improve adolescent health.

\section{ACKNOWLEDGEMENTS}

Past and current members of the Developmental Brain Imaging Laboratory are thanked for assisting in participant scheduling and data collection. Dr. Nagel and Dr. Morales received funding from the National Institute on Alcohol Abuse and Alcoholism (R01 AA017664; T32 AA07468).

\section{ADDITIONAL INFORMATION}

The online version of this article (https://doi.org/10.1038/s41386-018-0087-8) contains supplementary material, which is available to authorized users.

Competing interests: The authors declare no competing interests.

\section{REFERENCES}

1. Johnston LD, O'Malley PM, Miech RA, Bachman JG, Schulenberg JE. Monitoring the Future national survey results on drug use, 1975-2016: overview, key findings on adolescent drug use. Ann Arbor: Institute for Social Research, The University of Michigan; 2017. p. 113.

2. Spear LP. Adolescent alcohol exposure: are there separable vulnerable periods within adolescence? Physiol Behav. 2015;148:122-30.

3. Cheng HG, Anthony JC. Does our legal minimum drinking age modulate risk of first heavy drinking episode soon after drinking onset? Epidemiological evidence for the United States, 2006-2014. PeerJ. 2016;4:e2153.

4. Adam A, Faouzi M, Gaume J, Gmel G, Daeppen JB, Bertholet N. Age of first alcohol intoxication: association with risky drinking and other substance use at the age of 20. Swiss Med Wkly. 2011;141:w13226.

5. Hingson R, Heeren T, Zakocs R, Winter M, Wechsler H. Age of first intoxication, heavy drinking, driving after drinking and risk of unintentional injury among U.S. college students. J Stud Alcohol. 2003b;64:23-31.

6. Hingson R, Heeren T, Winter MR, Wechsler H. Early age of first drunkenness as a factor in college students' unplanned and unprotected sex attributable to drinking. Pediatrics. 2003a;111:34-41.

7. Xiao L, Bechara A, Grenard LJ, Stacy WA, Palmer P, Wei Y, et al. Affective decisionmaking predictive of Chinese adolescent drinking behaviors. J Int Neuropsychol Soc. 2009;15:547-57.

8. Goudriaan $A E$, Grekin ER, Sher KJ. Decision making and binge drinking: a longitudinal study. Alcohol Clin Exp Res. 2007;31:928-38.

9. Bjork JM, Pardini DA. Who are those "risk-taking adolescents"? Individual differences in developmental neuroimaging research. Dev Cogn Neurosci. 2015;11:56-64.

10. Galvan A, Hare TA, Parra CE, Penn J, Voss H, Glover G, et al. Earlier development of the accumbens relative to orbitofrontal cortex might underlie risk-taking behavior in adolescents. J Neurosci. 2006;26:6885-92.

11. Barkley-Levenson E, Galvan A. Neural representation of expected value in the adolescent brain. Proc Natl Acad Sci USA. 2014;111:1646-51.

12. Braams BR, van Duijvenvoorde AC, Peper JS, Crone EA. Longitudinal changes in adolescent risk-taking: a comprehensive study of neural responses to rewards, pubertal development, and risk-taking behavior. J Neurosci. 2015;35:7226-38.

13. Bunge SA, Wright SB. Neurodevelopmental changes in working memory and cognitive control. Curr Opin Neurobiol. 2007;17:243-50.

14. Durston S, Davidson MC, Tottenham N, Galvan A, Spicer J, Fossella JA, et al. A shift from diffuse to focal cortical activity with development. Dev Sci. 2006;9:1-8.

15. Mills KL, Goddings AL, Clasen LS, Giedd JN, Blakemore SJ. The developmental mismatch in structural brain maturation during adolescence. Dev Neurosci. 2014;36:147-60.

16. Casey BJ, Getz S, Galvan A. The adolescent brain. Dev Rev. 2008;28:62-77.
17. Jones SA, Cservenka A, Nagel BJ. Binge drinking impacts dorsal striatal response during decision making in adolescents. Neuroimage. 2016;129:378-88.

18. Cservenka A, Jones SA, Nagel BJ. Reduced cerebellar brain activity during reward processing in adolescent binge drinkers. Dev Cogn Neurosci. 2015;16:110-20.

19. Cservenka A, Nagel BJ. Risky decision-making: an FMRI study of youth at high risk for alcoholism. Alcohol Clin Exp Res. 2012;36:604-15.

20. Oldfield RC. The assessment and analysis of handedness: the Edinburgh inventory. Neuropsychologia. 1971;9:97-113.

21. Lucas CP, Zhang H, Fisher PW, Shaffer D, Regier DA, Narrow WE, et al. The DISC Predictive Scales (DPS): efficiently screening for diagnoses. J Am Acad Child Adolesc Psychiatry. 2001;40:443-9.

22. Brown SA, Myers MG, Lippke L, Tapert SF, Stewart DG, Vik PW. Psychometric evaluation of the Customary Drinking and Drug Use Record (CDDR): a measure of adolescent alcohol and drug involvement. J Stud Alcohol. 1998;59:427-38.

23. Wechsler D. Wechsler abbreviated scale of intelligence. San Antionio, TX: Psychological Corporation; 1999.

24. Hollingshead $A B$. Two factor index of social position. New Haven, CT: Privately Published; 1957.

25. Petersen AC, Crockett $L$, Richards $M$, Boxer A. A self-report measure of pubertal status: Reliability, validity, and initial norms. J Youth Adolesc. 1988;17:117-33.

26. Zuckerman M, Kuhlman DM, Joireman J, Teta P, Kraft M. A comparison of three structural models for personality: the big three, the big five, and the alternative five. J Pers Soc Psychol. 1993;65:757-68.

27. Ernst $M$, Nelson EE, McClure EB, Monk CS, Munson S, Eshel N, et al. Choice selection and reward anticipation: an fMRI study. Neuropsychologia. 2004:42:1585-97.

28. Sobell LC, Brown J, Leo Gl, Sobell MB. The reliability of the Alcohol Timeline Followback when administered by telephone and by computer. Drug Alcohol Depend. 1996;42:49-54.

29. National Institute on Alcohol Abuse and Alcoholism (2018). Drinking levels defined. https://www.niaaa.nih.gov/alcohol-health/overview-alcohol-consumption/moderatebinge-drinking. Online document.

30. Cox RW. AFNl: software for analysis and visualization of functional magnetic resonance neuroimages. Comput Biomed Res. 1996;29:162-73.

31. Cohen MS. Parametric analysis of fMRI data using linear systems methods. Neuroimage. 1997;6:93-103.

32. Siegel JS, Power JD, Dubis JW, Vogel AC, Church JA, Schlaggar BL, et al. Statistical improvements in functional magnetic resonance imaging analyses produced by censoring high-motion data points. Hum Brain Mapp. 2014;35:1981-96.

33. Cox RW, Chen G, Glen DR, Reynolds RC, Taylor PA. FMRI clustering in AFNI: falsepositive rates redux. Brain Connect. 2017;7:152-71.

34. Eklund $A$, Nichols TE, Knutsson $H$. Cluster failure: why $f M R I$ inferences for spatial extent have inflated false-positive rates. Proc Natl Acad Sci USA. 2016;113:7900-5.

35. Stopper CM, Floresco SB. Contributions of the nucleus accumbens and its subregions to different aspects of risk-based decision making. Cogn Affect Behav Neurosci. 2011;11:97-12.

36. Cardinal RN, Howes NJ. Effects of lesions of the nucleus accumbens core on choice between small certain rewards and large uncertain rewards in rats. BMC Neurosci. 2005;6:37.

37. Zalocusky KA, Ramakrishnan C, Lerner TN, Davidson TJ, Knutson B, Deisseroth $\mathrm{K}$ Nucleus accumbens D2R cells signal prior outcomes and control risky decisionmaking. Nature. 2016;531:642-6.

38. Buchel C, Peters J, Banaschewski T, Bokde AL, Bromberg U, Conrod PJ, et al. Blunted ventral striatal responses to anticipated rewards foreshadow problematic drug use in novelty-seeking adolescents. Nat Commun. 2017:8:14140.

39. Krain AL, Wilson AM, Arbuckle R, Castellanos FX, Milham MP. Distinct neural mechanisms of risk and ambiguity: a meta-analysis of decision-making. Neuroimage. 2006;32:477-84.

40. Litt $A$, Plassmann $H$, Shiv $B$, Rangel $A$. Dissociating valuation and saliency signals during decision-making. Cereb Cortex. 2011;21:95-102.

41. De Bellis MD, Wang L, Bergman SR, Yaxley RH, Hooper SR, Huettel SA. Neural mechanisms of risky decision-making and reward response in adolescent onset cannabis use disorder. Drug Alcohol Depend. 2013;133:134-45.

42. Kohno M, Nurmi EL, Laughlin CP, Morales AM, Gail EH, Hellemann GS, et al. Functional genetic variation in dopamine signaling moderates prefrontal cortical activity during risky decision making. Neuropsychopharmacology. 2016;41:695-703

43. Cohen MX, Young J, Baek JM, Kessler C, Ranganath C. Individual differences in extraversion and dopamine genetics predict neural reward responses. Brain Res Cogn Brain Res. 2005;25:851-61.

44. Krugel LK, Biele G, Mohr PN, Li SC, Heekeren HR. Genetic variation in dopaminergic neuromodulation influences the ability to rapidly and flexibly adapt decisions. Proc Natl Acad Sci USA. 2009;106:17951-6.

45. Muench C, Wiers CE, Cortes CR, Momenan R, Lohoff FW. Dopamine transporter gene methylation is associated with nucleus accumbens activation during reward 
1890

processing in healthy but not alcohol-dependent individuals. Alcohol Clin Exp Res. 2018:42:21-31.

46. Telzer EH, Ichien NT, Qu Y. Mothers know best: redirecting adolescent reward sensitivity toward safe behavior during risk taking. Soc Cogn Affect Neurosci. 2015;10:1383-91.

47. Chein J, Albert D, O'Brien L, Uckert K, Steinberg L. Peers increase adolescent risk taking by enhancing activity in the brain's reward circuitry. Dev Sci. 2011;14: F1-10
48. Tom SM, Fox CR, Trepel C, Poldrack RA. The neural basis of loss aversion in decision-making under risk. Science. 2007;315:515-8.

49. Alaux-Cantin S, Warnault V, Legastelois R, Botia B, Pierrefiche O, Vilpoux C, et al. Alcohol intoxications during adolescence increase motivation for alcohol in adult rats and induce neuroadaptations in the nucleus accumbens. Neuropharmacology. 2013;67:521-31. 